\title{
PRESENT STATUS OF RF SYSTEM FOR MEDICAL PROTON SYNCHROTRON*
}

\author{
Z. Fang", K. Endo, K. Egawa, S. Yamanaka, KEK, Tsukuba, Japan \\ T. Hirashima, T. Fusato, Y. Cho, Denki Kogyo Co., Aikou-gun, Japan
}

\section{Abstract}

The $200 \mathrm{MeV}$ proton synchrotron of circumference of $9.54 \mathrm{~m}$ is being developed for medical radiotherapy. The rf system has been carried out with a wide bandwidth of frequency sweeping from $2.0 \mathrm{MHz}$ to $17.8 \mathrm{MHz}$. The rf cavity is designed of a compact dimension and a high acceleration gradient. The high power test of the rf system has been successfully performed and maximal acceleration gradient of $60 \mathrm{kV} / \mathrm{m}$ has been achieved. The experiments with $\mathrm{rf}$ feedback control system tracking to the exciting current of bending magnets are being studied In this paper, the recent progress of the rf system and test results will be presented in detail.

\section{INTRODUCTION}

The compact proton synchrotron is being developed for the medical radiotherapy $[1,2,3]$. According to the lattice design of the machine, the proton synchrotron is operated in pulse mode with the beam acceleration time of $5 \mathrm{~ms}$, in which the bending magnet attains $3 \mathrm{~T}$ and the proton beam energy reaches $200 \mathrm{MeV}[4,5]$. The circumference of synchrotron is only $9.54 \mathrm{~m}$, so the beam acceleration system is required to have frequency sweeping from $2 \mathrm{MHz}$ to $17.8 \mathrm{MHz}$, and maximal rf cavity voltage of $10 \mathrm{kV}$, as shown in Fig. $1[6,7,8]$. Therefore, the key features of this $\mathrm{rf}$ system are the relatively wide bandwidth and the high acceleration gradient. The rf system has been designed and developed as shown in Fig. 2 , and the above requirements have been successfully achieved.

\section{RF CAVITY AND POWER AMPLIFIER}

The rf cavity consists of 2 cells with gaps located in the center of each cell. There are 4 high-permeability magnetic alloy cores loaded in each cell, and the cavity length is $400 \mathrm{~mm}$. The impedance at the two gaps has been measured. The average value of impedance of test result and the calculation result are shown in Fig. 3. It is shown that the test result agrees with the calculation result very well. The gap capacitance is about $24 \mathrm{pF}$, and the complex permeability of cores is $u=1288 f^{-0.81}-j 3161 f^{-0.81}$, where $f$ is in $\mathrm{MHz}$.

Two tetrode tubes 4CX35,000C are used to form a push-pull amplifier to drive the two cells of rf cavity in parallel, as shown in Fig. 4. All-pass network is applied to the input circuit for matching the tube's input capacitance. The parts of the all-pass network have been adjusted carefully, and SWR of tube input circuit is obtained smaller than 1.15 in the whole operation frequency range. A $2 \mathrm{~kW}$ preamplifier T145-6346B (Thamway Co., LTD) is used to drive the main amplifier.

\footnotetext{
*Work supported by MEXT of Japan

"fang@post.kek.jp
}

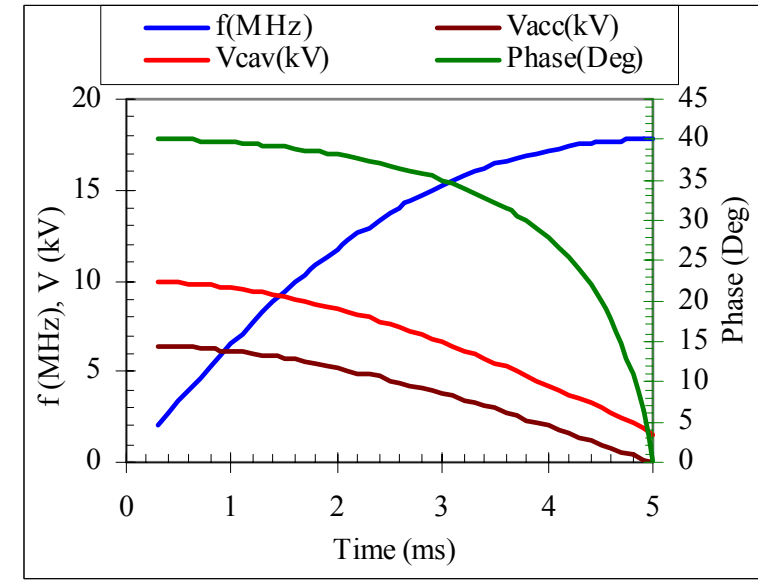

Figure 1: RF frequency, beam accelerating voltage, rf cavity voltage, and acceleration phase, as functions of acceleration time.

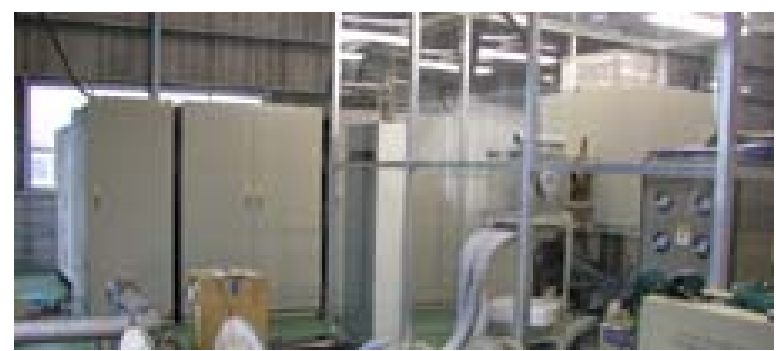

Figure 2: Developed rf system and power supply.

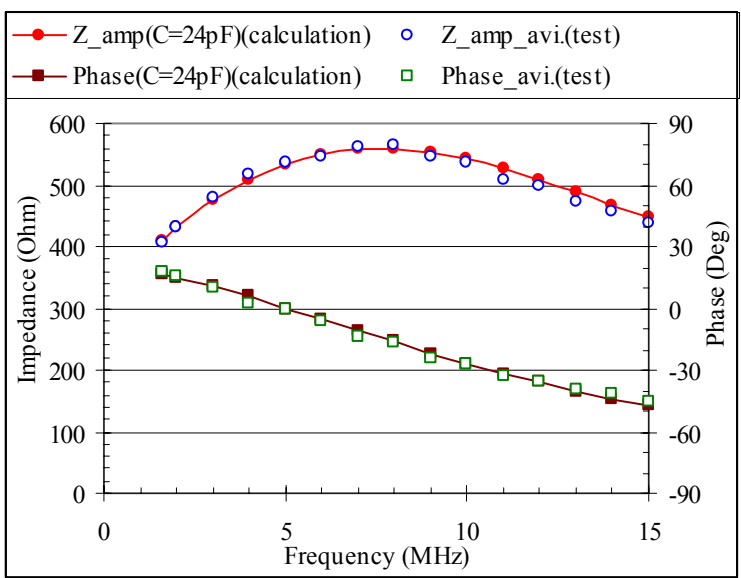

Figure 3: Test and calculation results of cavity gap impedance.

In order to improve the frequency response of $\mathrm{rf}$ system, an inductor $\left(L_{i}\right)$ of $1.9 \mu \mathrm{H}$ is inserted between the cavity and amplifier tube. The maximal acceleration gradient of $60 \mathrm{kV} / \mathrm{m}$ has been obtained. 


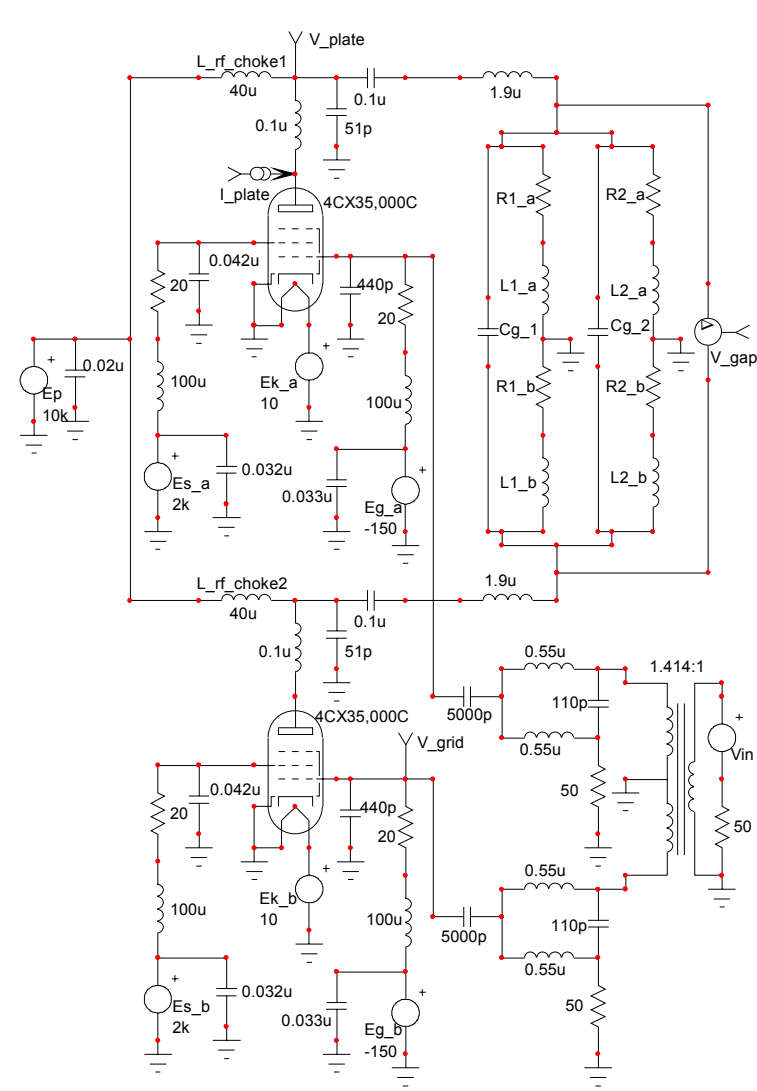

Figure 4: Circuit of rf cavity and power amplifier.

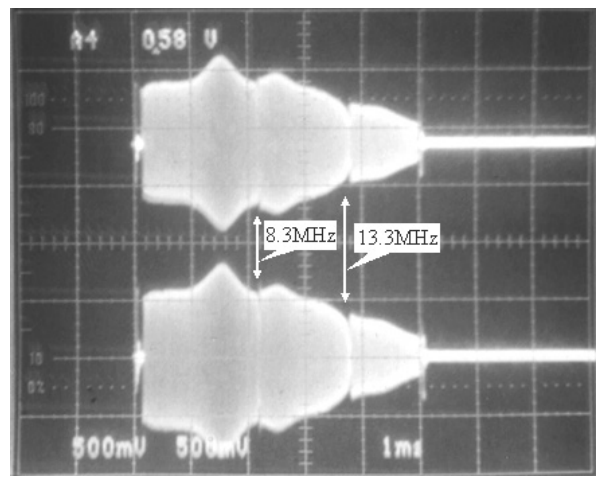

a) $160 \mu \mathrm{H}$

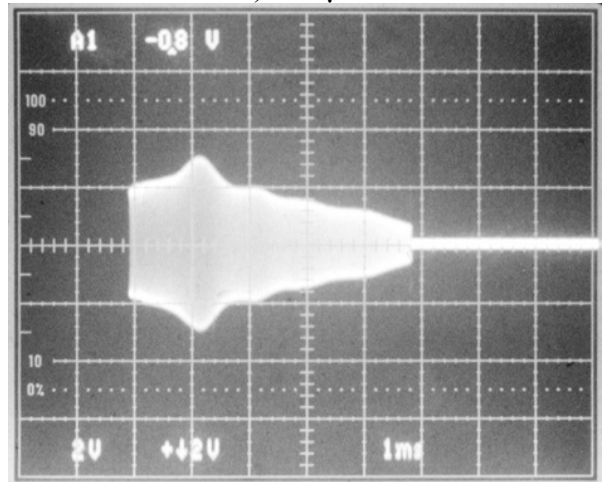

b) $40 \mu \mathrm{H}$

Figure 5: Waveform of $\mathrm{rf}$ cavity gap voltage with frequency sweeping from $2 \mathrm{MHz}$ to $18 \mathrm{MHz}$ for different $\mathrm{rf}$ choke.

\section{SOLUTION OF SELF-RESONANCES}

The original design of the anode rf choke for the rf system is $160 \mu \mathrm{H}$. However during the high power test, with input power increasing, arcing occurred in the anode rf choke around frequency of $8.3 \mathrm{MHz}$ and $13.3 \mathrm{MHz}$. The arcing is caused by the self-resonances due to the stray capacitance in the choke. So, we reduced the inductance of the rf choke from $160 \mu \mathrm{H}$ to $40 \mu \mathrm{H}$, and found the self-resonances are removed out completely finally. Table 1 shows the frequency of self-resonances in the rf choke. Fig. 5 shows the waveform of rf cavity gap voltage with frequency sweeping from $2 \mathrm{MHz}$ to $18 \mathrm{MHz}$ without feedback. The obtained maximal gap voltage is shown in Fig. 6. It shows the rf system can stably produce much higher voltage than required in the whole operation frequency region.

Table 1: Frequency of self-resonances in rf choke.

\begin{tabular}{|c|c|}
\hline $\mathrm{L}(\mu \mathrm{H})$ & $\mathrm{f}(\mathrm{MHz})$ \\
\hline 160 & $8.3,13.3$ \\
\hline 80 & 9 \\
\hline 60 & 15 \\
\hline 40 & No self-resonances \\
\hline
\end{tabular}

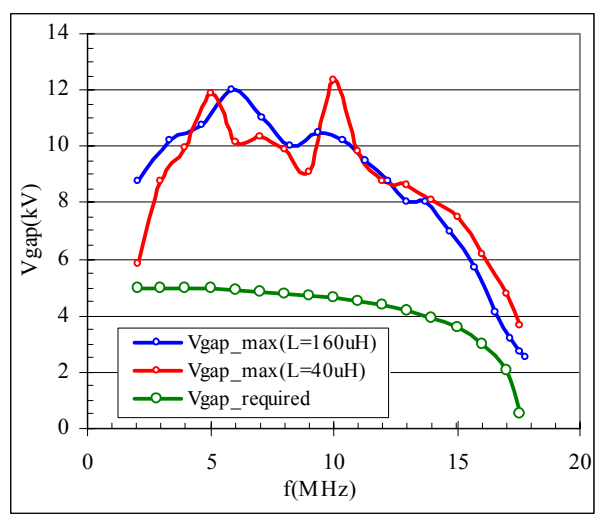

Figure 6: Obtained maximal and required gap voltages as functions of frequency.

\section{RF FEEDBACK CONTROL SYSTEM}

An rf feedback control system basing on a DDS signal generator has been developed as shown in Fig. 7. During the acceleration process, the rf frequency sweeps from $2 \mathrm{MHz}$ to $18 \mathrm{MHz}$, tracking to the exciting current of bending magnet. The envelope of $\mathrm{rf}$ gap voltage is $5 \cos (90 \pi \mathrm{t}) \mathrm{kV}$.

Two broadband high voltage probes for measuring gap voltage have been developed as shown in Fig. 8. The equivalent circuit of probe is shown in Fig. 9, where $\mathrm{C} 1$ is a coaxial capacitor. In order to reduce the effect of distributed inductance in large capacitor $\mathrm{C} 2$ and obtain a flat probe ratio, the capacitor $\mathrm{C} 2$ is divided into 6 parallel, and in each set, a capacitor of $470 \mathrm{pF}$ is connected with a resistor of $7.5 \mathrm{Ohm}$. The design ratio is around 2500 . The test result and fitting function of probe ratio is shown in Fig. 10, and the function is used in the rf control system for correction of cavity voltage. 


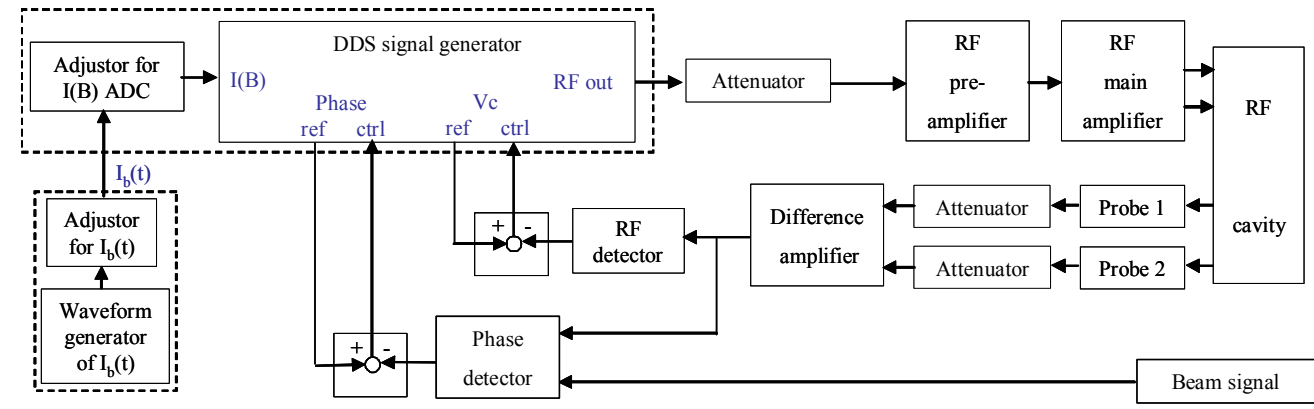

Figure 7: Block diagram of rf feedback control system.

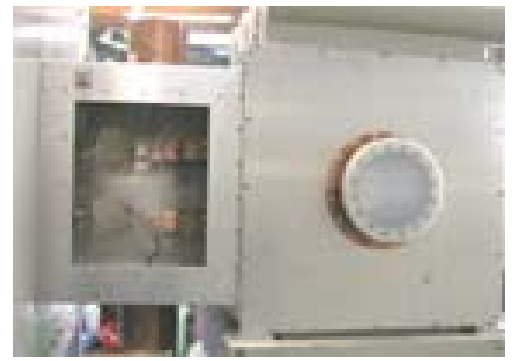

Figure 8: Developed broadband high voltage probes for rf cavity.

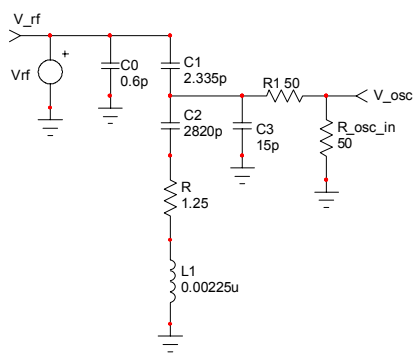

Figure 9: Circuit of broadband high voltage probe.

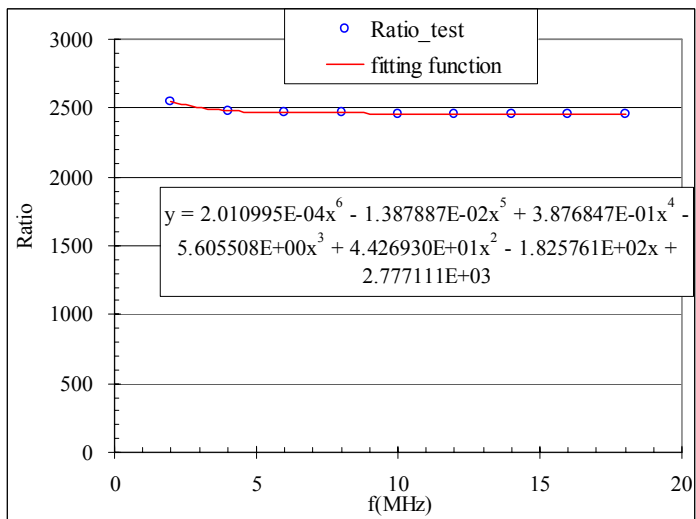

Figure 10: Test result and fitting function of probe ratio.

The experiments of rf control system have been successfully performed with feedback of gap voltage amplitude, as shown in Fig. 11. The green waveform (CH2) shows the rf cavity gap voltage with a cosine envelope and frequency sweeping from $2 \mathrm{MHz}$ to $18 \mathrm{MHz}$ tracking to the exciting current of bending magnet $(\mathrm{CH} 1)$.

\section{SUMMARY}

The rf system for the compact proton synchrotron has been developed. The high power test has been successfully performed to achieve the required gap voltage stably. And the experiments of rf feedback control system have been successfully carried out.

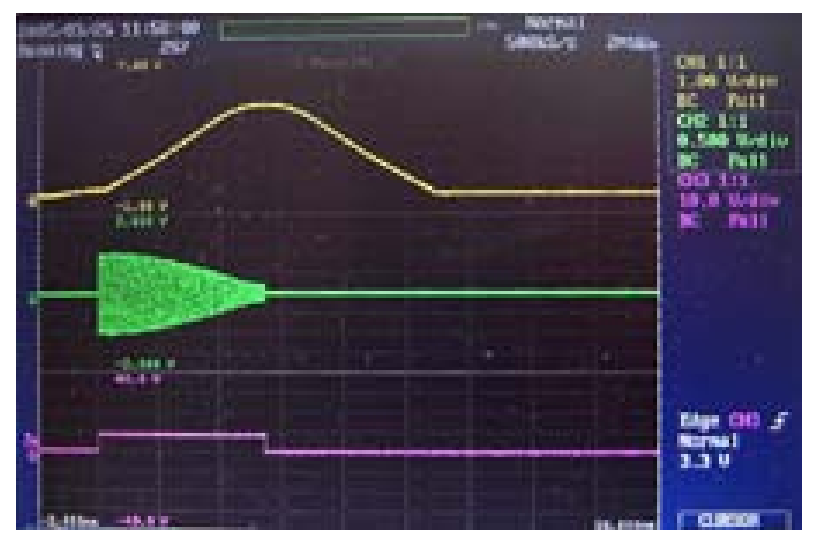

Figure 11: Waveforms of exciting current of bending magnet (CH1), gap voltage ( $\mathrm{CH} 2)$, and gate signal of $\mathrm{rf}$ system (CH3). (Time: $2 \mathrm{~ms} /$ div.)

\section{REFERENCES}

[1] K. Endo et al, "Development of High Field Dipole and High Current Pulse Power Supply for Compact Proton Synchrotron", PAC 2003, 1071-1073, USA.

[2] K. Endo et al, "High Field Pulse Dipole and Quadrupole Magnets for Compact Medical Pulse Synchrotron", APAC 2004, Korea.

[3] K. Endo et al, "Magnet and RF System of Small Pulse Synchrotron for Radiotherapy", EPAC 2004, 2661-2663, Switzerland.

[4] K. Endo et al, "Hardware Tracking Related to Compact Medical Pulse Synchrotron", PAC 2005, USA.

[5] S. Yamanaka et al, "Power Supply for Magnet of Compact Proton and/or Heavy ion Synchrotron for Radiotherapy", PAC 2005, USA.

[6] Z. Fang et al, "RF Cavities and Power Amplifier for the Compact Proton Synchrotron", PAC 2003, 1074-1076, USA.

[7] Z. Fang et al, "Test Results of RF Cavity and Power Amplifier for Compact Medical Proton Synchrotron", APAC 2004, Korea.

[8] Z. Fang et al, "RF System for Compact Medical Proton Synchrotron", EPAC 2004, 1039-1041, Switzerland. 